\title{
Morphological and Hemodynamic Differences Between Ruptured and Unruptured Vertebral Artery Dissecting Aneurysms: A Two-Center Retrospective Study
}

\author{
Heng Wei \\ Renmin Hospital of Wuhan University \\ Kun Yao \\ Renmin Hospital of Wuhan University \\ Qi Tian \\ Renmin Hospital of Wuhan University \\ Shoumeng Han \\ Renmin Hospital of Wuhan University \\ Wenhong Gao \\ Jinzhou Central Hospital \\ Wenrui Han \\ Renmin Hospital of Wuhan University \\ Sheng Liu \\ Renmin Hospital of Wuhan University \\ Guijun Wang \\ Renmin Hospital of Wuhan University \\ Qianxue Chen \\ Renmin Hospital of Wuhan University \\ Mingchang Li ( $\nabla$ mingcli@whu.edu.cn ) \\ Renmin Hospital of Wuhan University
}

\section{Research Article}

Keywords: computational fluid dynamic, morphological, hemodynamic, vertebral artery dissecting aneurysms, rupture risk

Posted Date: February 24th, 2022

DOI: https://doi.org/10.21203/rs.3.rs-1354471/v1 
License: (c) (i) This work is licensed under a Creative Commons Attribution 4.0 International License. Read Full License 


\section{Abstract \\ Purpose}

The morphological and hemodynamic features of patients with vertebral artery dissecting aneurysms (VADAs) are yet unknown. This study sought to elucidate morphological and hemodynamical features of patients with ruptured and unruptured VADAs.

\section{Methods}

This study recruited 31 patients with unruptured VADAs and 21 patients with ruptured VADAs who were treated at two hospitals. We collected data on their clinical, morphological, and hemodynamic characteristics. After reconstructing a patient-specific model, computational fluid dynamics (CFD) was performed. Binary logistic regression was used to analyze significant parameters and identify independent risk factors. Receiver operating characteristic (ROC) analysis identified cut-off values separating ruptured from unruptured aneurysms for each parameter.

\section{Results}

Three hemodynamic parameters were observed to be significantly different between ruptured and unruptured VADAs: intra-aneurysmal pressure (IAP), wall shear stress (WSS), and normalized pressure (NP). However, no morphological parameters had a significant difference between ruptured and unruptured VADAs. Binary logistic regression analysis revealed that IAP (AUC $=0.9662,95 \% \mathrm{Cl} 1.014-$ 1.068 ) and WSS (AUC $=0.7873,95 \% \mathrm{Cl} 1.124-16.859$ ) were independently significant variables in the hemodynamics model.

\section{Conclusion}

This study indicated significant hemodynamic differences but no significant morphological difference between ruptured and unruptured VADAs. Hemodynamic parameters of IAP and WSS are important in discriminating VADAs rupture status. These results should be confirmed through future larger studies.

\section{Introduction}

Vertebral artery dissecting aneurysms (VADAs) refer to a tear in the vertebral artery that results in an intramural hematoma and/or an aneurysmal dilatation, accounting for an average annual incidence rate of approximately $0.97-1.5$ cases per 100 population ${ }^{1}$. At present, the etiology of VADAs remains unclear, most literature believes that they occur spontaneously, while other reports speculate that a potentially fatal arterial injury causes them to occur during sports ${ }^{2,3}$. VADAs present with symptoms of posterior 
circulation ischemia due to vertebrobasilar artery ischemia and brain stem compression or with subarachnoid hemorrhage, resulting in high morbidity and mortality rates ${ }^{4}$. Therefore, some researchers suggest that early treatment of VADAs is necessary because rebleeding after rupture is very common ${ }^{5}$. Given the not-insignificant risk of treating unruptured VADAs as well as the known severe morbidity of aneurysm rupture, deciding on those aneurysms that require prophylactic treatment can be a quandary.

Numerous researches have been conducted on the risk of aneurysm rupture, but prediction accuracy remains not ideal ${ }^{6}$. There were some researches on the relationship between the morphological parameters and rupture risk of saccular aneurysms, but they have not been reported in VADAs. More recently, several publications have focused on the relationship between hemodynamics and IAs. Hemodynamics have been demonstrated to be crucial in development, growth, and rupture of saccular $\mid A s^{7}$. However, there are no report on hemodynamics with the rupture risk of VADAs.

This study aimed to identify morphological and hemodynamic risk factors of rupture among patients with VADAs to guide the treatment strategy of unruptured VADAs.

\section{Material And Methods}

\section{Protocol}

This study was approved by Clinical Research Ethics Committee of Renmin Hospital of Wuhan University, all methods were carried out in accordance with the guidelines and regulations for the Ethical Review of Biomedical Research involving Human beings issued by the National Health Commission of China in 2016. All the patients signed the informed consent form after admission and before operation.

\section{Patient selection}

All patient's data were retrospectively reviewed including imaging data and medical records from the electronic medical record system in two medical research centers. 52 consecutive patients who were diagnosed with VADAs were collected from March 2015 to May 2021. Cerebral Digital subtraction angiography (DSA) was used to diagnose all aneurysms. The clinical and serial imaging data of all patients were completed. Two neurosurgeons with over 10 years of experience in the interpretation of cerebral angiograms and the endovascular treatment of IAs confirmed the findings.

Inclusion criteria: (1) aneurysm located in the intracranial segment of the vertebral artery (V4 segment); (2) dissecting aneurysm. Exclusion criteria: (1) saccular aneurysms;(2) aneurysm located in the extracranial segment of the vertebral artery(V1-V3 segment); (3) patients with malignant tumors, malignant hypertension, and severe systemic disease; (4) combined with moyamoya disease or vascular malformations, traumatic aneurysms, multiple aneurysms; (5) abundant thrombosis in the sac of aneurysm; (6) 3D-DSA images with poor quality for CFD.

\section{Patient groups}


Patients were divided into rupture group and non-rupture group based on the presence of SAH. SAH were confirmed by computed tomography (CT) of brain and clinical condition. Lumbar puncture was considered mandatory to confirm the diagnosis in cases where SAH was suspected clinically but brain CT was negative. The results were confirmed by two neurosurgeons with over 10 years of experience in diagnosis and treatment of aneurysms.

\section{Imaging}

Standard transfemoral artery catheterization was used for all catheter angiographies. All 3D images were obtained through DSA using a Siemens Artis device (Siemens Healthineers , Forchheim, Germany), whereas rotational angiograms were performed $2 \mathrm{sec}$ after 5 -sec contrast injection, with a $18 \mathrm{ml}$ contrast agent at a rate of $3 \mathrm{ml} / \mathrm{s}$ and a $360^{\circ}$ rotation. The corresponding images were reconstructed into 3D modeling on the workstation and then exported in Digital Imaging and Communications in Medicine (DICOM) format.

\section{Aneurysm modeling}

DICOM format image data imported into the software Mimics medical 21.0 (Materialise, Leuven, Belgium) to segment geometries and obtain the preliminary 3D models, and then import into the software 3-matic medical 13.0 (Materialise, Leuven, Belgium ) in STL format for model repair and smoothing. Simulations were based on the finest grid with a maximum element size of $0.1 \mathrm{~mm}$, resulting in grids of roughly 7-12 million elements, dependent on particular visit model. The software ANSYS Fluent 2021 R2 and CFD-post 2021 R2 (ANSYS Inc.,USA) was used for simulations of hemodynamics and for generating the solution. The blood vessel was modelled as a rigid wall, and the blood flow was modelled as an incompressible Newtonian fluid with constant temperature and laminar flow, resulting in an NavierStokes equation approximate blood flow. A density of $1060 \mathrm{~kg} / \mathrm{m} 3$ and a dynamic viscosity of 0.0035 $\mathrm{Pa} / \mathrm{s}$ were specified for each simulation. A pulsatile Womersley velocity profile was prescribed at the inlets. Zero-pressure boundary conditions were set at all outlets. Finally, the calculation time was set to last for three cardiac cycles, with the result of the third cycle reaching a stable state after 200-time steps. Three pulsatile cycles were simulated to ensure that numerical stability had been reached, and the last cycle was taken as output. All data presented are time-averages over the third pulsatile cycle of flow simulation.

\section{Morphology analysis}

The 3D model of the aneurysm and parent vessels could be tumbled freely and measured on our workplace system, the observers found the best view angle to measure morphological variables. The morphological parameters used in this study including aneurysm maximum length (Lmax, maximum length of the aneurysm), aneurysm maximum width (Wmax, maximum diameter of the aneurysm), the average value of vessel diameters at the proximal aneurysm (Dp1) and vessel diameters at the distal aneurysm (Dp2), diameter of parent artery $(D p=(D p 1+D p 2) / 2)$. We also analyzed the following ratios related to the aneurysm shape, including L/ W ratio (Lmax / Wmax), Size ratio (SR). SR included SR1 
(Lmax/ Dp) and SR2 (Wmax/ Dp). The parameters were calculated by two Interventional physician who were blinded to the patient information and stability status. Discordance between the two evaluators was resolved by a third evaluator who has more than 10 years of experience in neuroradiology.

\section{Hemodynamic analysis}

Nine hemodynamic parameters defined on the aneurysm surface and volume used to characterize the aneurysm hemodynamics were calculated: WSS, IAP, normalized wall shear stress (NWSS), wall shear stress gradient (WSSG), Low shear area (LSA), oscillatory shear index (OSI), relative residence time (RRT), normalized pressure (NP), and combined hemodynamic parameters (CHP). WSS, WSSG, NWSS, IAP, OSI, NP, RRT and CHP were calculated the average value. WSS represents the pulling force acting tangential to the vascular wall; IAP is the force energy with which blood hits the inner wall of the aneurysm sac; NWSS represents the ratio of the aneurysm wall shear force to the mean value of the parent artery wall shear force; WSSG indicates the amplitude of variation along the wall shear force direction; LSA was described by the area of aneurysm wall exposed to the WSS below $10 \%$ of the mean WSS of the parent artery; OSI indicates the drastic change in the direction of wall shear force during a cardiac cycle; RRT characterizes the stagnation time of blood flow around the vascular wall; NP represents the ratio of the pressure in the aneurysm sac to the pressure in the parent artery and was used to compare the IAP of different aneurysms; CHP was the weighted average value of WSS and OSI. All the hemodynamic parameters were computed using the methods of previous reports ${ }^{8-10}$.

\section{Statistical analysis}

All data were analyzed using IBM SPSS Statistics version 25.0 (SPSS, Inc., Chicago, Illinois, US). Continuous variables were analyzed using independent t-test or Mann-Whitney $U$ test and are presented as means \pm SD or medians with interquartile range. Categorical variables are expressed as numbers and were analyzed using $\chi 2$ tests. Differences were considered statistically significant if the two-tailed $p$ values were $<0.05$ ( $95 \%$ confidence). Statistically significant variables in univariate analysis were further evaluated using binary logistic regression analysis to identify independent risk factors and ROC curves was performed to acquire cut off values.

\section{Results}

\section{Patient's clinical characteristics}

The total of 52 VADAs, 21 were ruptured and 31 were unruptured. The average age of ruptured group (12 males and 9 females) was $55.10 \pm 9.24$, and the average age of unruptured group (16 males and 15 females) was $53.74 \pm 12.68$. There was no significant difference in age, gender, smoking, drinking, hypertension, diabetes, CHD, hyperlipidemia between the two groups (Table 1). 
Table 1

Demographic information and clinical characteristics between ruptured and unruptured VADAs

\begin{tabular}{|c|c|c|c|}
\hline Variable & Ruptured & Unruptured & P-value \\
\hline Age & $55.10 \pm 9.24$ & $53.74 \pm 12.68$ & 0.677 \\
\hline \multicolumn{4}{|l|}{ Gender } \\
\hline Male & 12 & 16 & \multirow[t]{2}{*}{0.695} \\
\hline Female & 9 & 15 & \\
\hline \multicolumn{4}{|c|}{ Hypertension } \\
\hline Yes & 13 & 19 & \multirow[t]{2}{*}{0.964} \\
\hline No & 8 & 12 & \\
\hline \multicolumn{4}{|l|}{ Diabetes } \\
\hline Yes & 2 & 2 & \multirow[t]{2}{*}{0.683} \\
\hline No & 19 & 29 & \\
\hline \multicolumn{4}{|l|}{ CHD } \\
\hline Yes & 1 & 1 & \multirow[t]{2}{*}{0.777} \\
\hline No & 20 & 30 & \\
\hline \multicolumn{4}{|c|}{ hyperlipidemia } \\
\hline Yes & 2 & 5 & \multirow[t]{2}{*}{0.494} \\
\hline No & 19 & 26 & \\
\hline \multicolumn{4}{|l|}{ Drinking } \\
\hline Yes & 3 & 2 & \multirow[t]{2}{*}{0.347} \\
\hline No & 18 & 29 & \\
\hline \multicolumn{4}{|l|}{ Smoking } \\
\hline Yes & 5 & 6 & \multirow[t]{2}{*}{0.700} \\
\hline No & 16 & 25 & \\
\hline
\end{tabular}

\section{Morphologic Risk Factors Between Ruptured And Unruptured Vadas}


By comparing morphological parameters between ruptured VADAs and unruptured VADAs, we found that Wmax, Lmax, Dp1, Dp, SR1 and SR2 in the ruptured group were lower than the unruptured group, Dp2 and L/W higher than the unruptured group. However, all morphological parameters had no significant difference between the two groups (Table 2).

Table 2

Results of morphological characteristics between ruptured and unruptured VADAs

\begin{tabular}{|llll|}
\hline Variable & Ruptured & Unruptured & P-value \\
\hline Wmax & $7.171 \pm 3.306$ & $7.640 \pm 2.043$ & 0.530 \\
\hline Lmax & $10.075 \pm 3.105$ & $10.326 \pm 2.655$ & 0.756 \\
\hline Dp1 & $3.069 \pm 0.717$ & $3.175 \pm 0.436$ & 0.510 \\
Dp2 & $3.338 \pm 0.486$ & $3.322 \pm 0.472$ & 0.906 \\
\hline Dp & $3.173 \pm 0.524$ & $3.237 \pm 0.333$ & 0.589 \\
L/W & $1.516 \pm 0.402$ & $1.374 \pm 0.246$ & 0.119 \\
SR1 & $3.154 \pm 0.780$ & $3.167 \pm 0.817$ & 0.952 \\
\hline SR2 & $2.209 \pm 0.798$ & $2.342 \pm 0.616$ & 0.501 \\
\hline$* P<0.05$ was considered significant & \\
\hline
\end{tabular}

\section{Hemodynamic Risk Factors Between Ruptured And Unruptured Vadas}

Nine hemodynamic parameters of VADAs were analyzed between ruptured group and unruptured group (Fig. 1, Fig. 2). WSS, NP and IAP in ruptured group were significantly higher than unruptured group, while the other six hemodynamic parameters have no significant differences between the two groups (Table 3). 
Table 3

Results of hemodynamic characteristics of VADAs

\begin{tabular}{|llll|}
\hline Variable & Ruptured & Unruptured & P-value \\
\hline WSS & $2.822 \pm 1.198$ & $1.814 \pm 0.927$ & 0.001 \\
\hline NWSS & $0.486 \pm 0.159$ & $0.572 \pm 0.153$ & 0.055 \\
\hline WSSG & $123.174 \pm 27.088$ & $110.606 \pm 26.380$ & 0.102 \\
\hline NP & $1.393 \pm 0.088$ & $1.244 \pm 0.158$ & $<0.001$ \\
\hline OSI & $0.018 \pm 0.007$ & $0.020 \pm 0.008$ & 0.400 \\
\hline CHP & $0.163 \pm 0.043$ & $0.164 \pm 0.045$ & 0.952 \\
\hline RRT & $0.238 \pm 0.159$ & $0.217 \pm 0.142$ & 0.621 \\
\hline LSA & $0.339 \pm 0.157$ & $0.314 \pm 0.133$ & 0.533 \\
\hline IAP & $394.196 \pm 83.930$ & $205.920 \pm 66.551$ & $<0.001$ \\
\hline $\begin{array}{l}\text { VADAs, vertebral artery dissecting aneurysms; } \text { WSS, wall shear stress; } N W S S \text {, normalized wall shear } \\
\text { stress; } W S S G, \text { wall shear stress gradient; } N P \text {, normalized pressure; OSI, oscillatory shear index; } C H P, \\
\text { combined hemodynamic parameters; } R R T \text {, relative residence time; } \text { LSA, Low shear area; IAP, intra- } \\
\text { aneurysmal pressure. }{ }^{*} P<0.05 \text { was considered significant }\end{array}$ \\
\hline
\end{tabular}

\section{Binary Logistic Regression And Roc Analysis}

We performed binary logistic regression analysis on the three significant hemodynamic parameters of VADAs and found that IAP and WSS were independent predictive factors for the status of VADAs. The ORs indicated that the ruptured VADAs were 1.050 times $(95 \% \mathrm{Cl} 1.014$ to $1.086, p=0.006)$ more likely to be higher in IAP than unruptured VADAs, and 4.353 times $(95 \% \mathrm{Cl} 1.124$ to $16.859, p=0.033)$ (Table 4) more likely to be higher in WSS than unruptured VADAs.

Table 4

The results of binary logistic regression analysis

\begin{tabular}{|lll|}
\hline variable & OR $(95 \% \mathrm{Cl})$ & P-value \\
\hline WSS & $4.353(1.124,16.859)$ & 0.033 \\
\hline NP & $0.037(0.000,836.536)$ & 0.519 \\
\hline IAP & $1.050(1.014,1.086)$ & 0.006 \\
\hline WSS, wall shear stress; $N P$, normalized pressure; IAP, intra-aneurysmal pressure \\
\hline$* P<0.05$ was considered significant \\
\hline
\end{tabular}


The ROC analysis of independent predictors showed that IAP (AUC $=0.9662, p<0.001)$ and WSS (AUC = $0.7873, p<0.001)$ had diagnostic value for ruptured. The cut off values of IAP and WSS were $314.1 \mathrm{pa}$ and 2.597 pa (Fig. 3).

\section{Discussion}

In this study, by comparing eight morphological and nine hemodynamic parameters of VADAs, no morphological parameters were found to be associated with rupture status, whereas hemodynamic parameters IAP and WSS were linked to VADAs ruptured status. This is the first report to evaluate the rupture risk of VADAs according to hemodynamic parameters.

Unruptured VADAs are frequently attributed to sluggish growth patterns and often achieve good outcomes when medically managed, similarly to extracranial vascular dissections ${ }^{11}$. Ischemia occurred in approximately $77 \%$ of VADAs, with favorable outcomes in $82 \%^{12}$. However, in bleeding VADAs group, mortality rate was $20 \%$ in the treated group and $50 \%$ in the untreated group ${ }^{13,14}$. Because treatment involves a high risk of associated morbidity and mortality, appropriate treatments for unruptured VADAs remain controversial ${ }^{15}$. Therefore, it is very important to accurately predict the rupture risk of VADAs.

The morphological parameters of aneurysms are frequently thought to be associated with saccular aneurysm rupture. Previous studies have indicated that size, shape, location, irregularity, height-width ratio $(H / W)$, size ratio, and aspect ratio $(A R)$ of saccular aneurysms are all linked to rupture status ${ }^{16-19}$. However, the results of these studies were entirely morphological and concerned with saccular aneurysms. Fusiform or dissecting aneurysms were excluded in case selection of these studies. The present study examined the rupture risk of VADAs using eight morphological parameters and discovered that none of morphological parameters correlated with rupture status. These findings imply that it may be inappropriate to quantify the rupture risk of dissecting aneurysms using the common morphological factors previously utilized to calculate saccular aneurysms. Histologically, because media and adventitia of vertebral artery (VA) are weaker than those of internal carotid aneurysm, dissecting aneurysms are easily dilated between the two walls of VA, resulting in aneurysmal $\mathrm{SAH}^{20}$. Some people found that VADAs with pain are a critical predictor of stroke and a critical decision factor for surgical or endovascular treatment ${ }^{21,22}$. However, other studies have discovered that aggressive interventional treatment may not be necessary for patients with VADAs who only present with pain ${ }^{13,23}$. As a result, it is necessary to identify more precise predictive parameters suitable for predicting the rupture risk of dissecting aneurysms.

WSS is proposed as an important hemodynamic parameter of CFD-based studies. It represents a frictional force from blood flow tangential to the arterial lumen. Previous research has indicated a correlation between WSS and formation and rupture of aneurysms ${ }^{24-26}$. In this study, we discovered significantly higher mean WSS associated with rupture risk status of VADAs. Our findings corroborated those of prior research on saccular aneurysms were also applicable to VADAs. Aneurysm ruptured regions 
frequently occurred in thin-walled areas ${ }^{27,28}$. Previous studies revealed that thin regions of aneurysms have higher average WSS and IAP than hyperplastic regions. Moreover, ruptured aneurysms have significantly higher average and maximum WSS ${ }^{29,30}$. Ruptured aneurysms were characterized by more irregular shapes and were subjected to a more adverse hemodynamic environment, as described by faster flow, higher maximum WSS, higher mean WSS, etc. These associations with rupture status were consistent for different aneurysm locations ${ }^{31}$. Kim et al. also stated that aneurysms with thin areas had a higher WSS, despite their belief that a higher WSS alone might not provide a satisfactory characterization status of aneurysms ${ }^{32}$. Increased WSS inhibits the activation of endothelial cell receptors such as integrins and mechanically sensitive ion channel sensors, resulting in an increase in matrix metalloproteinase (MMP) secretion, causing degeneration of extracellular matrix (EMC) in vascular wall and injury of endothelial cells (ECs) ${ }^{33}$. When ECs layers are not damaged, WSS does not influence smooth muscle cells (SMCs). Once the damage occurred, SMCs initially respond to higher WSS by migrating from the media to the intima, and in response to local signals, they change from a contractile phenotype to a secretory type, increasing nuclear factor kappa beta (NF-K $\beta$ ), interleukin-1 beta (IL-1 $\beta$ ), tumor necrosis factor- $\alpha$ and matrix metals. Protease was produced, and the apoptotic pathway was initiated $^{34}$. IL-1 $\beta$ was found to locally act on SMCs in mouse aneurysm model, resulting in apoptosis of SMCs, weakening of elasticity layer, and aneurysm rupture ${ }^{35}$. Increased WSS stimulates SMC migration and phenotypic alterations, resulting in secreting inflammatory mediators and factors involved in vascular wall degradation, hence encouraging aneurysm rupture.

IAP is the kinetic energy of blood transferred as an inertial force perpendicular to the aneurysms wall surface. The local pressure increases at a flow impingement point as fluid kinetic energy is converted to static wall pressure ${ }^{36}$. Previous research has examined changes in IAP following endovascular embolization with stent implantation for aneurysms and the risk of postoperative rupture. Piasecki et al. found that stent implantation resulted in a significant decrease in IAP $(p=0.046)$ during diastolic. Systolic or mean IAP did not differ significantly ${ }^{37}$. Yu et al. also found that stent implantation significantly lowered IAP and WSS in a reconstructed model ${ }^{38}$. IAP was previously measured by inserting a microwire probe such as an arterial pressure transducer into the lumen of an aneurysm, and no significant difference was observed between ruptured and unruptured aneurysms. This measurement yields a value comparable to that of the parent artery pressure ${ }^{39}$. A study has indicated an obvious linear relationship between aneurysm pressure and radial artery blood pressure ${ }^{40}$. Therefore, we believe that there may be obvious errors in IAP results measured by this method. In our present study, IAP represents the energy perpendicular to the aneurysm wall caused by the impact of blood flow on the aneurysm wall, and it is also vertical to the tension direction represented by WSS ${ }^{41}$. It is intimately linked to velocity and blood flow state ${ }^{42}$. Our findings indicate a statistically significant difference in IAP between ruptured and unruptured VADAs, and the AUC $=96.62 \%(p<0.001)$. This finding suggest that it may be a more appropriate parameter for predicting VADAs rupture risk. 
There were some potential limitations in our study. First, although we selected patients from two centers for the study, the number of cases remains small due to low incidence of VADAs, which may have a deviation from the trial's results. Additional multi-center studies with larger samples are required to corroborate our results. Second, while there are several studies on morphological parameters in saccular aneurysms but few on dissecting aneurysms, morphological parameters we selected may not be comprehensive or precise. Third, this study was a retrospective analysis, using a patient-specific model in CFD simulation, but the entrance boundary conditions were not patient-specific, and the hemodynamic simulation employed assumptions of laminar flow, Newtonian blood, and rigid wall. This may lead to inaccurate trial results. Fourth, some studies demonstrated that morphology of ruptured aneurysms may change ${ }^{43}$. This may possibly have occurred in hemodynamics before and after aneurysm rupture $e^{44,45}$.

\section{Conclusion}

We compared eight morphological and nine hemodynamic parameters between ruptured and unruptured VADAs. We discovered no morphological parameters that can discriminants of VADAs rupture status. Meanwhile, WSS and IAP were found as independently significant variables, which clinically useful discriminants of VADAs rupture status with high AUC values. This shows that there is a correlation between hemodynamics and the ruptured status of VADAs. The ability of WSS and IAP to predict rupture risk need to be assessed in large prospective studies that include follow up of patients with unruptured VADAs.

\section{Declarations}

Funding information The authors disclosed receipt of the following financial support for the research, authorship, and publication of this article: This research was funded by National Natural Science Foundation of China (No. 81971870 and No. 82172173).

Conflict of interest disclosure The authors declare no competing interests or financial ties to disclose.

Author contributions H.W., K.Y., W.G., Q.C and M.L. contributed the conception, design and drafted the manuscript. H.W., Q.T., S.H., W.H., S.L., G.W. contributed data acquisition and analysis, H.W., K.Y., Q.C. and M.L. made the Article preparation, editing and review. All authors contributed to the article and approved the submitted version.

Data availability statement The raw data supporting the conclusions of this article will be made available by the authors, without undue reservation.

\section{References}

1. Pomeraniec, I. J., Mastorakos, P., Raper, D. \& Park, M. S. Rerupture Following Flow Diversion of a Dissecting Aneurysm of the Vertebral Artery: Case Report and Review of the Literature. World Neurosurg 143, 171-179, doi:10.1016/j.wneu.2020.07.149 (2020). 
2. Choi, M. H. et al. Preferential location for arterial dissection presenting as golf-related stroke. AJNR Am J Neuroradiol 35, 323-326, doi:10.3174/ajnr.A3768 (2014).

3. Suzuki, S. et al. Traumatic vertebral artery dissection in high school rugby players: A report of two cases. Journal of Clinical Neuroscience 47, 137-139, doi:10.1016/j.jocn.2017.10.008 (2018).

4. Guan, J. et al. Endovascular treatment for ruptured and unruptured vertebral artery dissecting aneurysms: a meta-analysis. J Neurointerv Surg 9, 558-563, doi:10.1136/neurintsurg-2016-012309 (2017).

5. Naito, I., Iwai, T. \& Sasaki, T. Management of intracranial vertebral artery dissections initially presenting without subarachnoid hemorrhage. Neurosurgery 51 (2002).

6. Mangrum, W. I. et al. Enlarging vertebrobasilar nonsaccular intracranial aneurysms: frequency, predictors, and clinical outcome of growth. J Neurosurg 102, 72-79, doi:10.3171/jns.2005.102.1.0072 (2005).

7. Meng, H., Tutino, V. M., Xiang, J. \& Siddiqui, A. HighWSS or LowWSS? Complex Interactions of Hemodynamics with Intracranial Aneurysm Initiation, Growth, and Rupture: Toward a Unifying Hypothesis. AJNR Am J Neuroradiol 35, 1254-1262, doi:10.3174/ajnr.A3558 (2014).

8. Xiang, J. et al. Hemodynamic-morphologic discriminants for intracranial aneurysm rupture. Stroke 42, 144-152, doi:10.1161/strokeaha.110.592923 (2011).

9. Jou, L. D., Lee, D. H., Morsi, H. \& Mawad, M. E. Wall shear stress on ruptured and unruptured intracranial aneurysms at the internal carotid artery. AJNR Am J Neuroradiol 29, 1761-1767, doi:10.3174/ajnr.A1180 (2008).

10. Yuan, J. et al. Hemodynamic and Morphological Differences Between Unruptured Carotid-Posterior Communicating Artery Bifurcation Aneurysms and Infundibular Dilations of the Posterior Communicating Artery. Front Neurol 11, 741, doi:10.3389/fneur.2020.00741 (2020).

11. Urasyanandana, K. et al. Treatment outcomes in cerebral artery dissection and literature review. Interventional Neuroradiology 24, 254-262, doi:10.1177/1591019918755692 (2018).

12. Arnold, M. et al. Vertebral artery dissection: presenting findings and predictors of outcome. Stroke 37, 2499-2503, doi:10.1161/01.STR.0000240493.88473.39 (2006).

13. Wang, J. et al. Endovascular management of vertebrobasilar artery dissecting aneurysms. Turk Neurosurg 23, 323-328, doi:10.5137/1019-5149.Jtn.6513-12.1 (2013).

14. Rabinov, J. D., Hellinger, F. R., Morris, P. P., Ogilvy, C. S. \& Putman, C. M. Endovascular management of vertebrobasilar dissecting aneurysms. AJNR Am J Neuroradiol 24, 1421-1428 (2003).

15. Park, S. I. et al. Clinical and angiographic follow-up of stent-only therapy for acute intracranial vertebrobasilar dissecting aneurysms. AJNR Am J Neuroradiol 30, 1351-1356, doi:10.3174/ajnr.A1561 (2009).

16. Xu, W. D. et al. Morphology parameters for rupture in middle cerebral artery mirror aneurysms. J Neurointerv Surg 12, 858-861, doi:10.1136/neurintsurg-2019-015620 (2020). 
17. Xu, Z., Kim, B. S., Lee, K. S., Choi, J. H. \& Shin, Y. S. Morphological and clinical risk factors for the rupture of posterior communicating artery aneurysms: significance of fetal-type posterior cerebral artery. Neurol. Sci. 40, 2377-2382, doi:10.1007/s10072-019-03991-4 (2019).

18. Cui, Y. et al. Aneurysm morphological prediction of intracranial aneurysm rupture in elderly patients using four-dimensional CT angiography. Clin Neurol Neurosurg 208, 106877, doi:10.1016/j.clineuro.2021.106877 (2021).

19. Ludwig, C. G., Lauric, A., Malek, J. A., Mulligan, R. \& Malek, A. M. Performance of Radiomics derived morphological features for prediction of aneurysm rupture status. J Neurointerv Surg 13, 755-761, doi:10.1136/neurintsurg-2020-016808 (2021).

20. Yamaura, A., Ono, J. \& Hirai, S. Clinical picture of intracranial non-traumatic dissecting aneurysm. Neuropathology: official journal of the Japanese Society of Neuropathology 20, 85-90, doi:10.1046/j.1440-1789.2000.00276.x (2000).

21. Jin, S. C., Kwon, D. H., Choi, C. G., Ahn, J. S. \& Kwun, B. D. Endovascular strategies for vertebrobasilar dissecting aneurysms. AJNR Am J Neuroradiol 30, 1518-1523, doi:10.3174/ajnr.A1621 (2009).

22. Shin, Y. S., Kim, H. S. \& Kim, S. Y. Stenting for vertebrobasilar dissection: a possible treatment option for nonhemorrhagic vertebrobasilar dissection. Neuroradiology 49, 149-156, doi:10.1007/s00234006-0169-x (2007).

23. Kobayashi, N. et al. Natural course of dissecting vertebrobasilar artery aneurysms without stroke. AJNR Am J Neuroradiol 35, 1371-1375, doi:10.3174/ajnr.A3873 (2014).

24. Salimi Ashkezari, S. F. et al. Hemodynamics in aneurysm blebs with different wall characteristics. J Neurointerv Surg, doi:10.1136/neurintsurg-2020-016601 (2020).

25. Huang, Z. et al. A Hemodynamic Mechanism Correlating with the Initiation of MCA Bifurcation Aneurysms. AJNR Am J Neuroradiol 41, 1217-1224, doi:10.3174/ajnr.A6615 (2020).

26. Neyazi, B. et al. Rupture risk assessment for multiple intracranial aneurysms: why there is no need for dozens of clinical, morphological and hemodynamic parameters. Therapeutic Advances in Neurological Disorders 13, doi:10.1177/1756286420966159 (2020).

27. Suzuki, T. et al. Determining the Presence of Thin-Walled Regions at High-Pressure Areas in Unruptured Cerebral Aneurysms by Using Computational Fluid Dynamics. Neurosurgery 79, 589-595, doi:10.1227/NEU.0000000000001232 (2016).

28. Jiang, P. et al. Hemodynamic characteristics associated with thinner regions of intracranial aneurysm wall. Journal of clinical neuroscience: official journal of the Neurosurgical Society of Australasia 67, 185-190, doi:10.1016/j.jocn.2019.06.024 (2019).

29. Cebral, J. R. et al. Local Hemodynamic Conditions Associated with Focal Changes in the Intracranial Aneurysm Wall. American Journal of Neuroradiology 40, 510-516, doi:10.3174/ajnr.A5970 (2019).

30. Furukawa, K. et al. Hemodynamic characteristics of hyperplastic remodeling lesions in cerebral aneurysms. PLoS ONE 13, e0191287, doi:10.1371/journal.pone.0191287 (2018).

31. Detmer, F. J. et al. Associations of hemodynamics, morphology, and patient characteristics with aneurysm rupture stratified by aneurysm location. Neuroradiology 61, 275-284, 
doi:10.1007/s00234-018-2135-9 (2019).

32. Kim, J. H. et al. Hemodynamic Features of Microsurgically Identified, Thin-Walled Regions of Unruptured Middle Cerebral Artery Aneurysms Characterized Using Computational Fluid Dynamics. Neurosurgery 86, 851-859, doi:10.1093/neuros/nyz311 (2020).

33. Hoi, Y. et al. In vivo assessment of rapid cerebrovascular morphological adaptation following acute blood flow increase.

34. Appelboom, G. et al. Role of the complement cascade in cerebral aneurysm formation, growth, and rupture. Neuroimmunology and Neuroinflammation 2, doi:10.4103/2347-8659.154888 (2015).

35. Chalouhi, N. et al. Biology of intracranial aneurysms: role of inflammation. J Cereb Blood Flow Metab 32, 1659-1676, doi:10.1038/jcbfm.2012.84 (2012).

36. Jeong, W. \& Rhee, K. Hemodynamics of Cerebral Aneurysms: Computational Analyses of Aneurysm Progress and Treatment. Comput Math Methods Med 2012, 782801, doi:10.1155/2012/782801 (2012).

37. Piasecki, P., Ziecina, P., Brzozowski, K., Wierzbicki, M. \& Narloch, J. Intra-aneurysmal pressure changes during stent-assisted coiling. PLoS ONE 15, e0233981, doi:10.1371/journal.pone.0233981 (2020).

38. Yu, H., Huang, G. P., Yang, Z. \& Ludwig, B. R. Numerical studies of hemodynamic alterations in preand post-stenting cerebral aneurysms using a multiscale modeling. Int $\mathrm{J}$ Numer Method Biomed Eng 35, e3256, doi:10.1002/cnm.3256 (2019).

39. Li, Y., Corriveau, M., Aagaard-Kienitz, B., Ahmed, A. \& Niemann, D. Differences in Pressure Within the Sac of Human Ruptured and Nonruptured Cerebral Aneurysms. Neurosurgery 84, 1261-1268, doi:10.1093/neuros/nyy182 (2019).

40. Hasan, D. M., Hindman, B. J. \& Todd, M. M. Pressure changes within the sac of human cerebral aneurysms in response to artificially induced transient increases in systemic blood pressure. Hypertension 66, 324-331, doi:10.1161/HYPERTENSIONAHA.115.05500 (2015).

41. Staarmann, B., Smith, M. \& Prestigiacomo, C. J. Shear stress and aneurysms: a review. Neurosurg Focus 47, E2, doi:10.3171/2019.4.FOCUS19225 (2019).

42. Shojima, M. et al. Role of the bloodstream impacting force and the local pressure elevation in the rupture of cerebral aneurysms. Stroke 36, 1933-1938, doi:10.1161/01.STR.0000177877.88925.06 (2005).

43. Amenta, P. S. et al. Analysis of nonmodifiable risk factors for intracranial aneurysm rupture in a large, retrospective cohort. Neurosurgery 70, 693-699; discussion 699-701, doi:10.1227/NEU.0b013e3182354d68 (2012).

44. Cornelissen, B. M. W. et al. Hemodynamic Differences in Intracranial Aneurysms before and after Rupture. AJNR Am J Neuroradiol 36, 1927-1933, doi:10.3174/ajnr.A4385 (2015).

45. Sejkorova, A. et al. Hemodynamic changes in a middle cerebral artery aneurysm at follow-up times before and after its rupture: a case report and a review of the literature. Neurosurg Rev 40, 329-338, doi:10.1007/s10143-016-0795-7 (2017). 


\section{Figures}

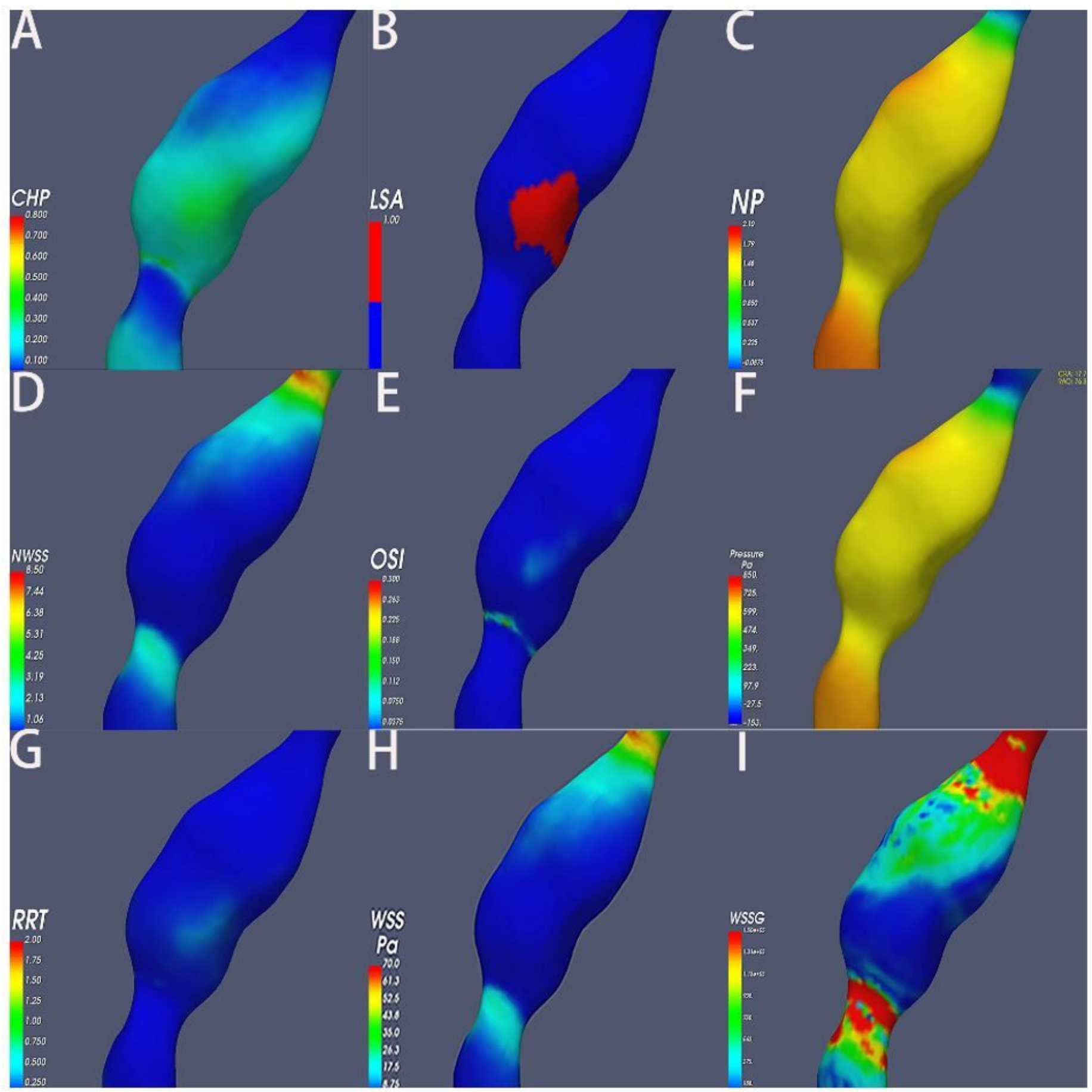

\section{Figure 1}

Hemodynamic analysis of ruptured VADAs. (A) combined hemodynamic parameters (CHP). (B) Low shear area (LSA). (C) normalized pressure (NP). (D) normalize wall shear stress (NWSS). (E) oscillatory shear index (OSI). (F) intra-aneurysmal pressure (Pressure, IAP). (G) relative residence time (RRT). (H) wall shear stress (WSS). (I) wall shear stress gradient (WSSG). 


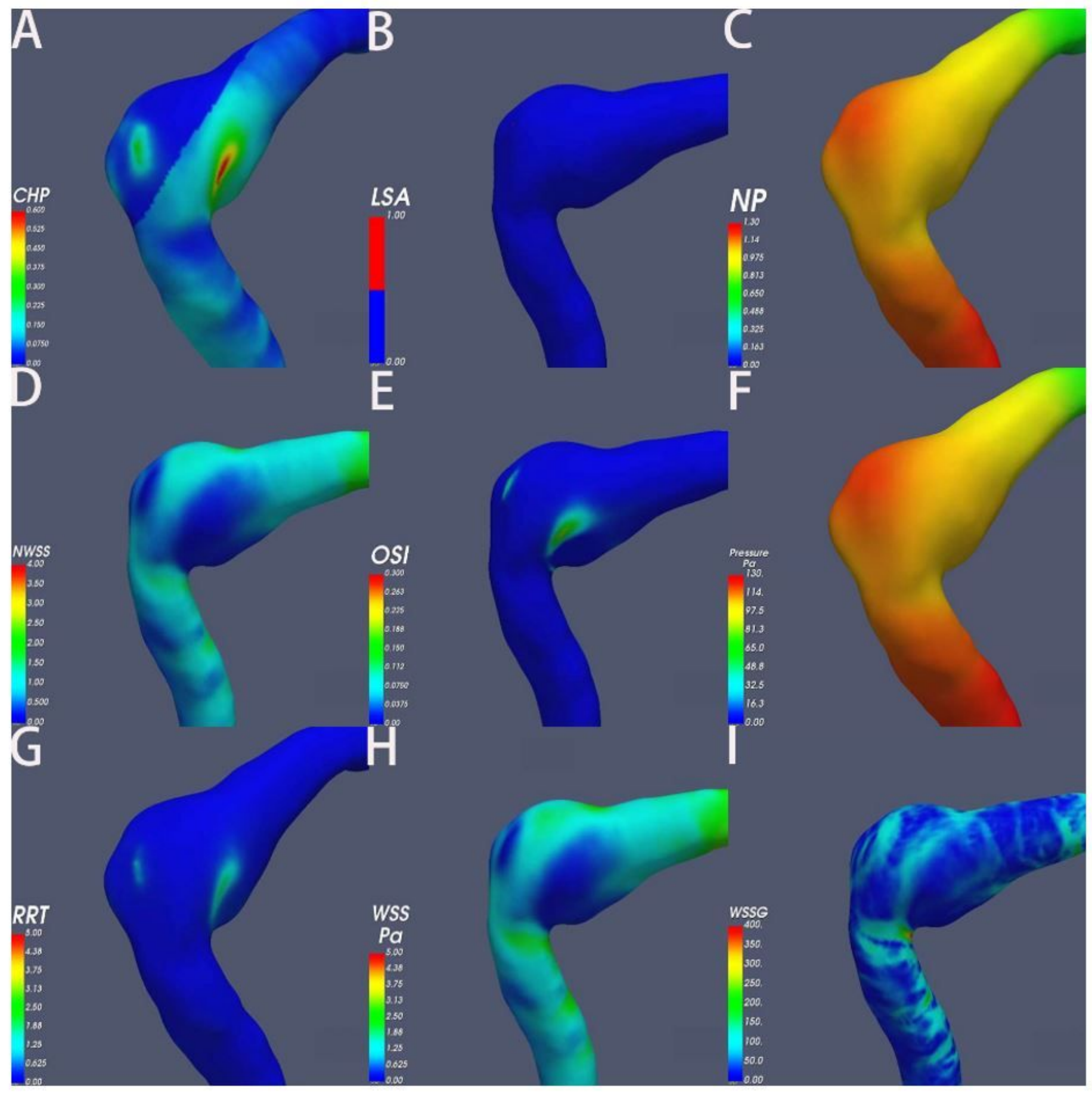

Figure 2

Hemodynamic analysis of unruptured VADAs. (A) combined hemodynamic parameters (CHP). (B) Low shear area (LSA). (C) normalized pressure (NP). (D) normalize wall shear stress (NWSS). (E) oscillatory shear index (OSI). (F) intra-aneurysmal pressure (Pressure, IAP). (G) relative residence time (RRT). (H) wall shear stress (WSS). (I) wall shear stress gradient (WSSG). 

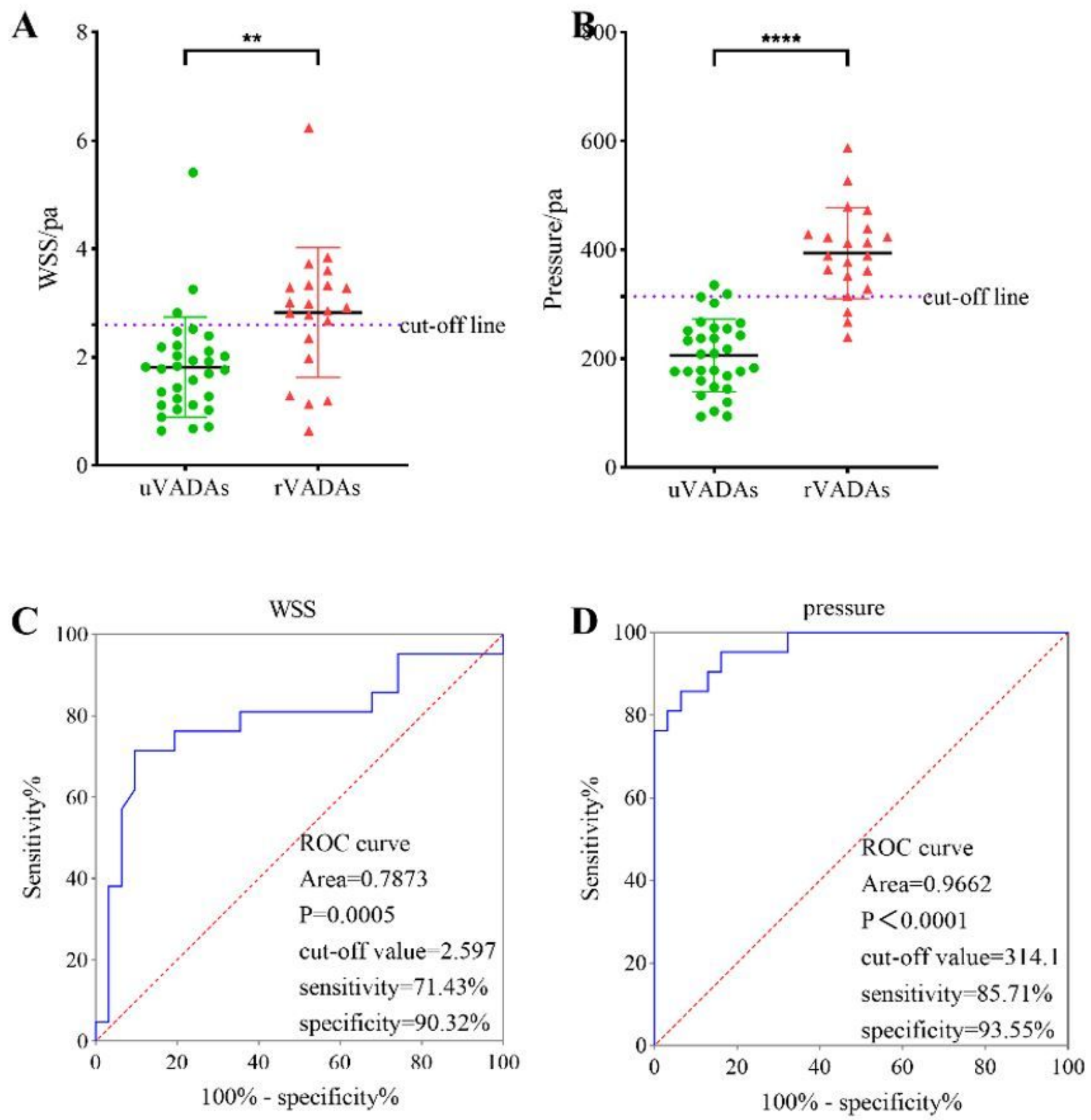

Figure 3

ROC curves for independent risk factors of WSS and IAP 